\title{
Elaboración de un snack expandido de haba (Vicia faba 1.)
}

\author{
Elaboration of an expanded bean snack (Vicia faba L.) \\ Calvimontes V. Adriana'; Cortez V., Gladys²; Huaringa J. Amelia ${ }^{3}$
}

\begin{abstract}
Resumen
En el presente trabajo se evaluó cuál de las cinco variedades de haba (Vicia faba L.): Amarilla, Blanca, Gergona, Señorita y Verde, era la más adecuada para elaborar un snack, mediante el proceso de expansión por explosión, teniendo como variable la humedad de ingreso al proceso de expansión (12\% y 15\%), manteniendo una presión constante de 276 $\mathrm{kPa}$. Se realizó un análisis proximal de cada variedad y se determinó el contenido y composición del almidón así como el porcentaje de cáscara, luego de lo cual las muestras fueron acondicionadas (peladas y ajustadas a la humedad inicial requerida) para ingresar al proceso de expansión, realizado en un cañón de expansión. Posteriormente, al producto obtenido se le determinó el índice de expansión (IE), el porcentaje de humedad, el rendimiento de la expansión y del proceso. Finalmente, se realizó la evaluación sensorial; mediante una prueba Ranking se determinó la variedad de haba de mayor aceptación; con una Categorización Cuantitativa se caracterizaron las variedades expandidas y mediante un Perfil de Textura se compararon las características del haba expandida con las de un haba frita comercial. Se determinó que el haba de la variedad Blanca fue la más adecuada para el proceso y que el contenido de humedad antes de ingresar al proceso afecta el índice de expansión, siendo la humedad de $12 \%$ la que da mayores valores de IE. Comparando el haba frita comercial con el haba expandida se encontró que existen diferencias significativas en cuanto a su condición de crocante, adhesividad y gomosidad, pero no en dureza, superando en ser crocante al haba frita comercial, con la ventaja de tener un menor contenido de grasa.
\end{abstract}

Palabras clave: expansión por explosión; haba, snack; expandido; perfil de textura.

\begin{abstract}
In this research we evaluated which of five cultivars of beans (Vicia faba L.): Amarilla, Blanca, Gergona, Señorita and Verde, was the most suitable for the elaboration of a snack through the expansion process. The variable in study was humidity prior the expanded process (12\% and 15\%), with a constant pressure of $276 \mathrm{kPa}$. A proximal analysis of each cultivar was carried out to determine the amount and composition of starch of the varieties, after that the samples were conditioned (by peeling and adjusting the initial moisture to the required values) before entering the process of expansion, which takes place in an expansion barrel. After that the product was evaluated in its physical and chemical properties: expansion index (EI), percentage of humidity and the performance of the process and expansion. Finally, a sensorial analysis was performed, through a Ranking Test we determined the bean cultivar with the greatest acceptance, then with a Quantitative Categorization we characterized the expanded cultivars, and using a Texture Profile we compared the characteristics of the expanded bean with a commercial fried bean. The final conclusions were that the Blanca cultivar was the most suitable for the process, and also that the humidity of the bean before the process affects the expansion index, being $12 \%$ of humidity the one that generates the highest IE values. When comparing the commercial fried bean with the expanded bean, there were significant differences regarding its crispiness, adhesiveness and stickiness, but there were no differences when it came to hardness, being crispier than the commercial fried bean and with the advantage of having a lower fat content.
\end{abstract}

Keywords: explosion expansion; bean; expanded; snack; texture profile.

\section{Introducción}

El haba (Vicia faba L.) es una leguminosa con un alto valor nutricional, ya que posee un promedio de $25 \%$ de proteínas, además de vitaminas $\mathrm{A}, \mathrm{B}_{1} \mathrm{~B}_{2} \mathrm{y}$ minerales como el fósforo y el potasio que la hacen una de las preferidas en la dieta del poblador andino (Huaringa, A., Camarena, F., Chiappe, L. y Mostacero, E., 2003) y una buena alternativa nutricional, habiéndose duplicado su producción de 31,9 miles de toneladas en la campaña 1996 - 1997 a 69,5 miles de toneladas en la campaña 2008 - 2009 (MINAG, 2010). De otro lado, el nivel de desnutrición crónica en niños menores de cinco años es uno de los principales problemas socioeconómicos del Perú, teniéndose el más alto nivel de desnutrición en la sierra $(27,6 \%)$ seguido

\footnotetext{
1 Facultad de Ingeniería, Universidad Nacional Agraria La Molina, Lima (Perú). Email: a_calvimontes@hotmail.com

2 Universidad Nacional Agraria La Molina, Lima (Perú). Email: g_cortez@lamolina.edu.pe

3 Universidad Nacional Agraria La Molina, Lima (Perú). Email: ahuaringa@lamolina.edu.pe
} 
de la selva $(21,7 \%)$ y la costa $(8,4 \%)$. Los departamentos que presentan mayores índices de desnutrición crónica son Huancavelica $(44,7 \%)$, Cajamarca $(32 \%)$, Huánuco (31\%), Apurímac (30,9\%), Ayacucho (30,3\%) y Cusco (29,1\%), según datos del INEI, 2011. Dado el elevado costo de la proteína de origen animal, es de suma importancia dar a conocer las fuentes vegetales de proteínas de menor costo, además de buscar alternativas de procesamiento para darles un valor agregado, en especial en las zonas rurales donde se producen.

La tecnología de expansión es muy conocida y empleada en cereales como el trigo, maíz y arroz y sus requerimientos son reducidos, lo cual la hace adecuada para zonas rurales. Sin embargo, aún no se ha probado esta tecnología en leguminosas, por tanto se hace necesaria la determinación adecuada de los parámetros para este proceso.

El presente trabajo de investigación busca una alternativa de procesamiento del haba que permita su mayor consumo, en especial en la zona rural y en los lugares con mayor desnutrición infantil. De esta forma, las comunidades campesinas que se dedican al cultivo de habas podrían dirigir sus esfuerzos hacia la siembra y cosecha de una de estas variedades, cuya transformación industrial sería de bajo costo y de fácil implementación. Por ello, los objetivos de la investigación son: 1) Caracterizar fisicoquímicamente cinco variedades de haba (Vicia faba L.); 2) Determinar la variedad de haba más adecuada para la elaboración de un snack expandido de habas y 3) Evaluar el producto expandido fisicoquímica y sensorialmente.

\section{Materiales y métodos}

El proceso de expansión se realizó en un local comercial ubicado en el distrito de Comas. Los análisis fisicoquímicos se realizaron en el laboratorio de Biotecnología de la Facultad de Industrias Alimentarias, el Laboratorio de Química de la Facultad de Ciencias y en el Laboratorio de Evaluación Nutricional de Alimentos de la Facultad de Zootecnia en la Universidad Nacional Agraria La Molina (UNALM). Como materia prima se tuvieron

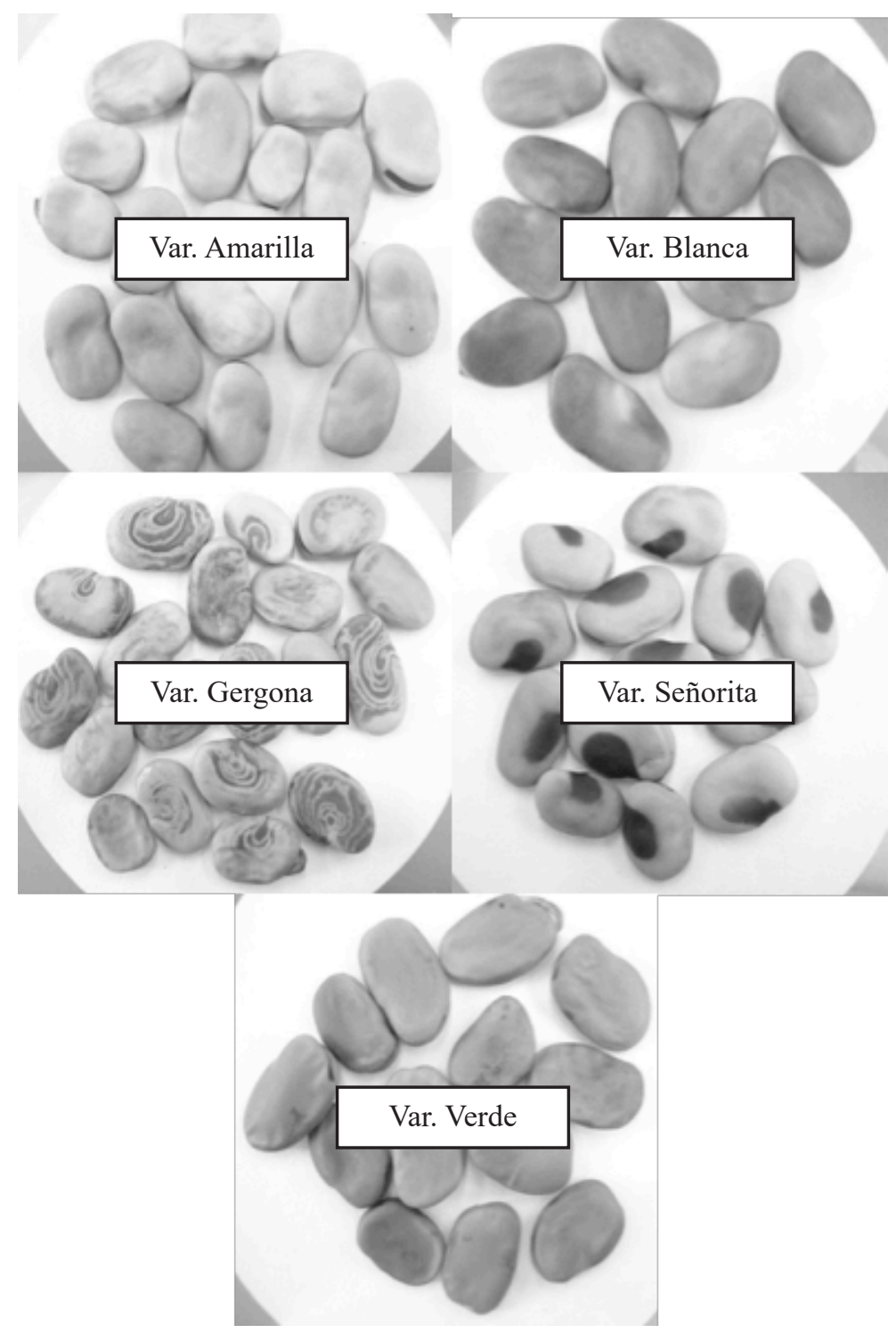

Figura 1. Variedades de habas evaluadas. 
cinco variedades de habas secas (Fig. 1): la variedad Amarilla, proveniente del Programa de Investigación de Leguminosas de grano de la UNALM, y las variedades Blanca, Verde, Gergona y Señorita, procedentes del Instituto Nacional de Investigación Agraria (INIA), Estación Experimental Santa Ana de Huancayo.

Se empleó un cañón de expansión (fabricación nacional) de hierro fundido, de $1,5 \mathrm{~kg}$ de capacidad, con motor eléctrico y soplete a gas.
El diseño estadístico se realizó en dos partes; en la primera se evaluó un Diseño Completamente al Azar (DCA) para determinar si el índice de expansión era estadísticamente diferente a las humedades de $12 \%$ y $15 \%$ iniciales; mediante una prueba de Kruskall Wallis y con la prueba de Correlación de Pearson se determinó si la composición de las habas (proteína, carbohidratos, almidón y amilopectina) estaba asociada al índice de expansión. En la segunda parte se evaluó un Diseño de Bloques Completamente al Azar (DBCA), donde cada variedad de haba se consideró

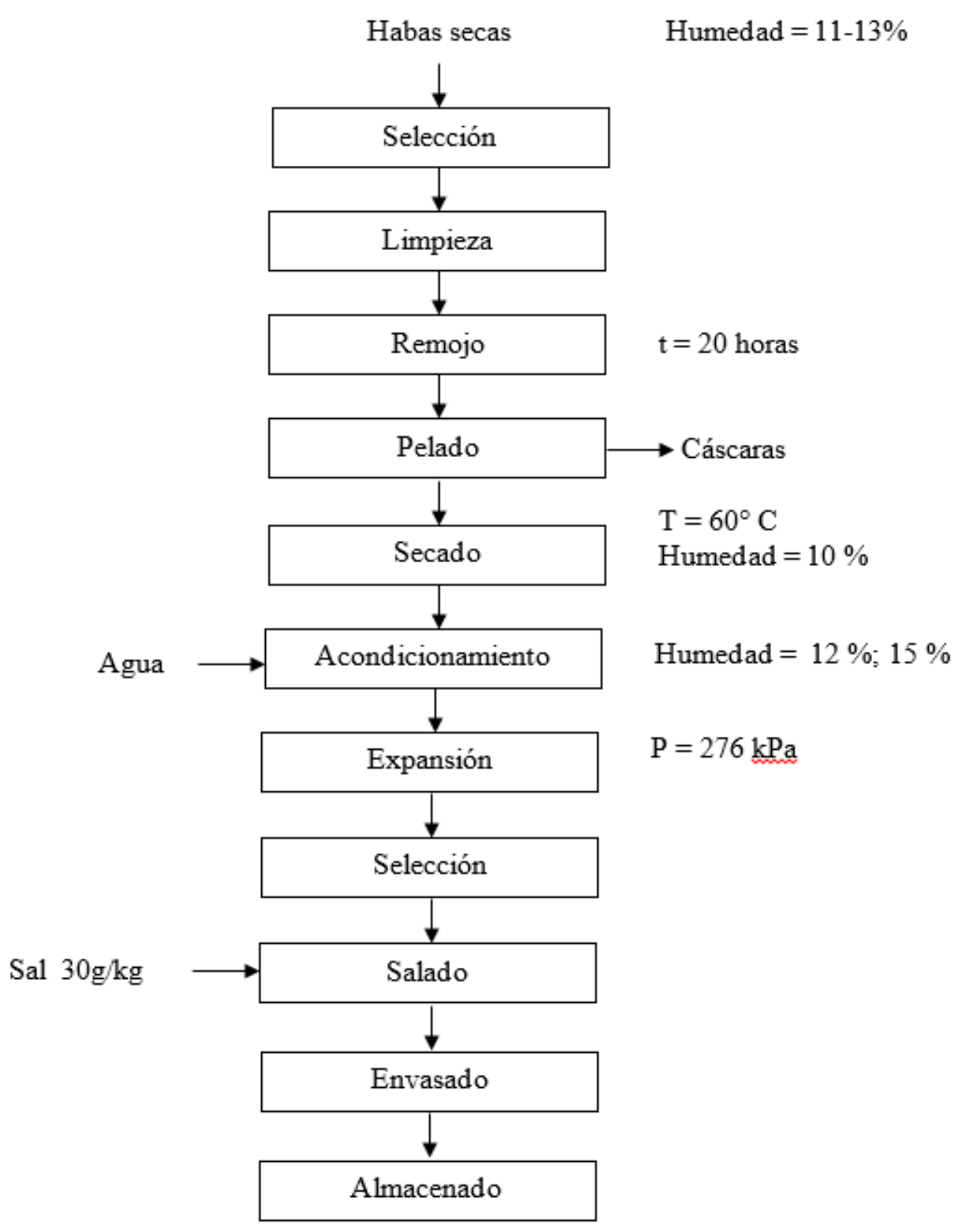

Figura 2. Flujo de operaciones para la elaboración de un snack de habas expandidas. 
como un bloque y los datos obtenidos fueron analizados con las pruebas estadísticas de Duncan y Friedman como alternativa no paramétrica.

Para el análisis sensorial, en la prueba de Ranking se determinó la variedad más aceptada por el consumidor; los datos fueron procesados mediante la prueba no paramétrica de Friedman y, para determinar si existían diferencias significativas entre la aceptación de las diferentes variedades de habas procesadas a $12 \%$ de humedad inicial, se realizó una prueba de Categorización Cuantitativa Relativa (en escala no estructurada) para las características de sabor, olor, color y textura. Los datos acumulados para las cuatro características fueron procesados con un DBCA en un Análisis de Variancia (ANVA), utilizando la prueba $\mathrm{F}$ de Snedecory y, por último, se realizó un Perfil de Textura para comparar la variedad elegida como ganadora con un haba frita comercial.

\section{Metodología}

En la Fig. 2 se presenta el flujo de operaciones para la elaboración del snack expandido de habas, el cual consta de lo siguiente:

- Selección: Se eliminaron las habas que no cumplían con las características de tamaño e inocuidad requeridas (presencia de hongos, enfermedades de la semilla, insectos, etc.).

- Lavado: Se realizó un lavado con la finalidad de eliminar el polvo e impurezas.

- Remojo: Se colocaron las habas secas en tinas con agua durante una noche (20 horas) para facilitar el pelado.

- Pelado: Se realizó un pelado manual y se separaron las cáscaras para ser cuantificadas.

- Secado: Se realizó en un secador de bandejas a una temperatura de $60^{\circ} \mathrm{C}$, hasta llegar a una humedad de $10 \%$.

- Acondicionamiento: Los granos fueron humectados con un sistema de spray utilizando cálculos de balance de masa hasta llegar a las humedades requeridas de $12 \%$ y $15 \%$ para cada variedad de habas.

- Expansión: Se organizaron contenedores con $1 \mathrm{~kg}$ de la muestra acondicionada; se calentó el equipo (30 min.) hasta que la presión interna del cañón fuera de $276 \mathrm{kPa}$ para poder luego retirar el soplete y abrir la compuerta.
- Selección: Se seleccionaron las habas expandidas manualmente, desechando los pedazos rotos y las habas quemadas.

- Salado: se espolvoreó sal mientras el producto estuvo caliente para facilitar la adhesión.

- Envasado: Se envasaron las habas expandidas y saladas en bolsas de polipropileno y se sellaron con calor.

- Almacenado: se almacenaron las bolsas selladas en un lugar fresco y seco.

\section{Método de Análisis \\ Análisis de la materia prima}

El análisis químico-proximal se realizó según la metodología indicada en La asociación de las comunidades analíticas (AOAC, 1995), la determinación del contenido de almidón según Soni et al. (citado en Cenzano, 1996) y la determinación del porcentaje de amilosa y amilopectina según Mc Grance, Cornell y Melbourne (1998).

\section{Análisis del producto final}

El análisis químico-proximal se realizó según le metodología indicada en el AOAC (1995), el índice de expansión se calculó según la fórmula: $\mathrm{IE}=$ Volumen final/Volumen inicial (Capcha, 1977) y la determinación del color según el manual del colorímetro Konica Minolta (2003). Para la evaluación sensorial, se realizó una prueba Ranking y una Categorización Cuantitativa Relativa según Ureña, D’Arrigo y Girón (1999) y Perfil de Textura según Núñez (2009).

\section{Resultados y discusión \\ De la materia prima}

\section{Análisis químico - proximal}

La composición química de las cinco variedades de habas enteras utilizadas se presenta en la Tabla 1. Comparando los resultados obtenidos con los de Huaringa, Camarena, Chiappe y Mostacero (2003), se encontraron valores de proteína cercanos, siendo la variedad con mayor contenido de proteína el Haba Señorita $(25,40 \%)$ y la de menor valor el Haba Verde $(22,92 \%)$.

El Haba Blanca presentó el mayor contenido de grasa

Tabla 1. Composición químico-proximal de las cinco variedades de habas. (g/100 g en bh).

\begin{tabular}{lccccc}
\hline \multicolumn{1}{c}{ Componente } & $\begin{array}{c}\text { Var. } \\
\text { Amarilla }\end{array}$ & $\begin{array}{c}\text { Var. } \\
\text { Blanca }\end{array}$ & $\begin{array}{c}\text { Var. } \\
\text { Gergona }\end{array}$ & $\begin{array}{c}\text { Var. } \\
\text { Señorita }\end{array}$ & Var. Verde \\
\hline Humedad (\%) & 13,18 & 11,39 & 12,68 & 12,16 & 11,53 \\
Carbohidratos (\%) & 50,68 & 53,45 & 52,83 & 49,74 & 52,43 \\
Proteína (\%) & 24,59 & 23,39 & 22,76 & 25,40 & 22,92 \\
Grasa (\%) & 1,48 & 1,63 & 1,30 & 1,52 & 1,41 \\
Fibra (\%) & 6,39 & 6,12 & 6,70 & 7,17 & 7,67 \\
Ceniza (\%) & 3,68 & 4,02 & 3,73 & 4,01 & 4,04 \\
\hline
\end{tabular}


Tabla 2. Contenido de carbohidrato, almidón, amilosa y amilopectina en las habas evaluadas.

\begin{tabular}{lcccc}
\hline Variedad & $\begin{array}{c}\text { Porcentaje de } \\
\text { Carbohidratos (en } 100 \mathrm{~g})\end{array}$ & $\begin{array}{c}\text { Porcentaje de } \\
\text { Almidón (b.s.) }\end{array}$ & $\begin{array}{c}\text { Porcentaje de } \\
\text { Amilosa (b.s.) }\end{array}$ & $\begin{array}{c}\text { Porcentaje de } \\
\text { Amilopectina (b.s.) }\end{array}$ \\
\hline Haba Amarilla & 50,68 & 14,53 & 46,51 & 53,49 \\
Haba Blanca & 53,45 & 12,70 & 46,51 & 53,49 \\
Haba Gergona & 52,83 & 11,43 & 45,02 & 54,98 \\
Haba Señorita & 49,74 & 16,78 & 44,14 & 55,86 \\
Haba Verde & 52,43 & 14,49 & 44,97 & 55,03 \\
\hline
\end{tabular}

$(1,63 \%)$ y carbohidratos $(53,45 \%)$, mientras que el Haba Verde presentó un alto contenido de fibra $(7,67 \%)$ y ceniza $(4,04 \%)$. Al comparar la composición del grano de haba con otros cereales usados comúnmente en la expansión de granos, se encontró que estas variedades tienen valores mayores de proteína $(22,76 \%-25,4 \%)$ que la kiwicha, la cual contiene $15 \%$-22\% de este componente, según Tosi et al. (citado en Zapotoczny, Markowski, Majewska, Ratajski y Konopko, 2004).

En cuanto al producto final, se encontró que no existe una correlación estadística entre los componentes y los índices de expansión obtenidos, según la evaluación de Correlación de Pearson, por lo que no se puede asociar el contenido de carbohidratos o proteínas con el índice de expansión.

\section{Contenido de almidón, amilosa y amilopectina}

Los resultados de estos contenidos se pueden apreciar en la Tabla 2. Las variedades de habas evaluadas presentaron un menor contenido de carbohidratos que los cereales utilizados en la tecnología de expansión por explosión como el arroz, maíz y trigo que tienen un contenido de 85 $\%, 70 \%$ y $60 \%$ respectivamente (Callejo, 2002), siendo el máximo valor entre las habas el de la Blanca $(53,45 \%)$.

Un contenido de $55 \%$ a $70 \%$ de amilosa dará como resultado un producto expandido crocante $\mathrm{y}$ fuerte mientras que un mayor valor de amilopectina dará un producto con mayor expansión y por lo tanto será más crujiente. Todas las variedades de habas presentaron un contenido de amilosa menor a $50 \%$ y un mayor contenido de amilopectina, por lo que el snack de haba expandida tendría capacidad de expandirse y ser un snack más crujiente que duro; sin embargo el máximo valor de amilopectina fue de la variedad Señorita $(55,86 \%)$, la cual no excedió de $56 \%$, por lo que no se aprecia el mismo comportamiento de expansión que en un cereal.

Tabla 3. Porcentaje de cáscara de los granos de habas

\begin{tabular}{|l|c|}
\hline Variedad & \% Cáscara \\
\hline Haba Amarilla & 20,67 \\
\hline Haba Blanca & 24,76 \\
\hline Haba Gergona & 18,95 \\
\hline Haba Señorita & 25,22 \\
\hline Haba Verde & 19,81 \\
\hline
\end{tabular}

\section{Porcentaje de cáscara}

En la Tabla 3 se presenta el porcentaje de cáscara; este fue eliminado antes de realizar el proceso de expansión, facilitando el acondicionamiento de la humedad.

El Haba Señorita presentó el mayor porcentaje de cáscara, alcanzando un 25,22 \%, mientras el Haba Gergona presentó el menor porcentaje de las cinco variedades, con 18,95\%. La cáscara constituye gran parte de los desechos y puede ser reutilizada como componente de abonos (compost), lo cual es una ventaja y una oportunidad si se elabora el snack en zonas rurales. El porcentaje de cáscara afectará el rendimiento total del proceso.

\section{Del producto terminado \\ Índice de expansión}

Las habas expandidas no mostraron una porosidad homogénea como las que suelen presentar los cereales expandidos. En vez de tener pequeñas burbujas distribuidas en su interior, presentaron una gran burbuja en el centro del cotiledón (Fig. 3). Esto se debe a que el contenido de amilosa y amilopectina en los productos expandidos, extruidos y fritos, influye en la textura final (Lusas y Rooney, 2001). En la Tabla 4 se compara el contenido de amilosa y amilopectina con el IE respectivo de cada muestra. Se encontró que hay diferencias significativas en el índice de expansión con $12 \%$ de humedad inicial y con $15 \%$, siendo la humedad que mejor IE dio la de $12 \%$ y las variedades con mayor expansión fueron las

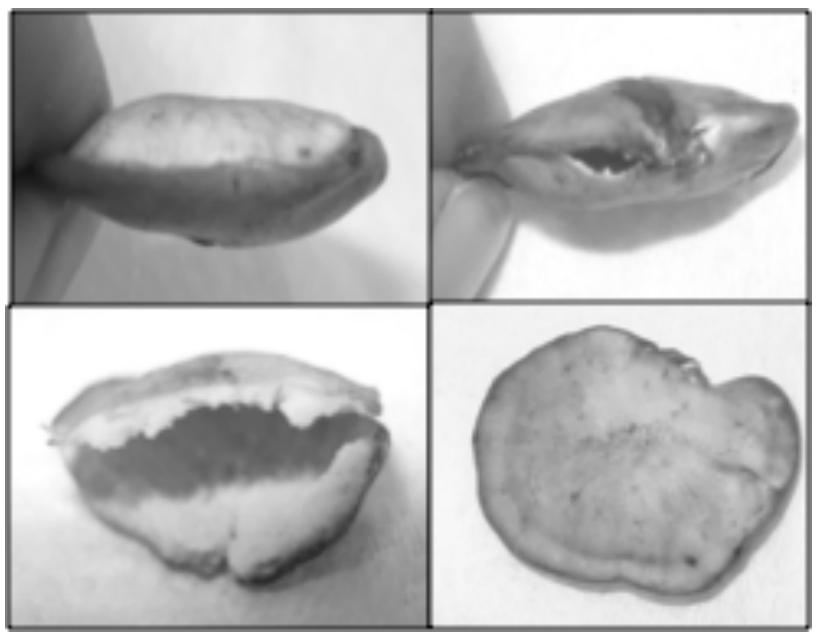

Figura 3. Haba expandida. 
Tabla 4. Comparación del contenido y composición del almidón contra el índice de expansión de las habas evaluadas

\begin{tabular}{lcccc}
\hline \multicolumn{1}{c}{ Variedad } & Porcentaje de Amilosa & $\begin{array}{c}\text { Porcentaje de } \\
\text { Amilopectina }\end{array}$ & $\begin{array}{c}\text { Índice de expansión } \\
(12 \%)\end{array}$ & $\begin{array}{c}\text { Índice de expansión } \\
(15 \%)\end{array}$ \\
\hline Haba Amarilla & 46,51 & 53,49 & 1,07 & 1,00 \\
Haba Blanca & 46,51 & 53,49 & 1,31 & 1,13 \\
Haba Gergona & 45,02 & 54,98 & 1,40 & 1,08 \\
Haba Señorita & 44,14 & 55,86 & 1,39 & 1,21 \\
Haba Verde & 44,97 & 55,03 & 1,06 & 1,01 \\
\hline
\end{tabular}

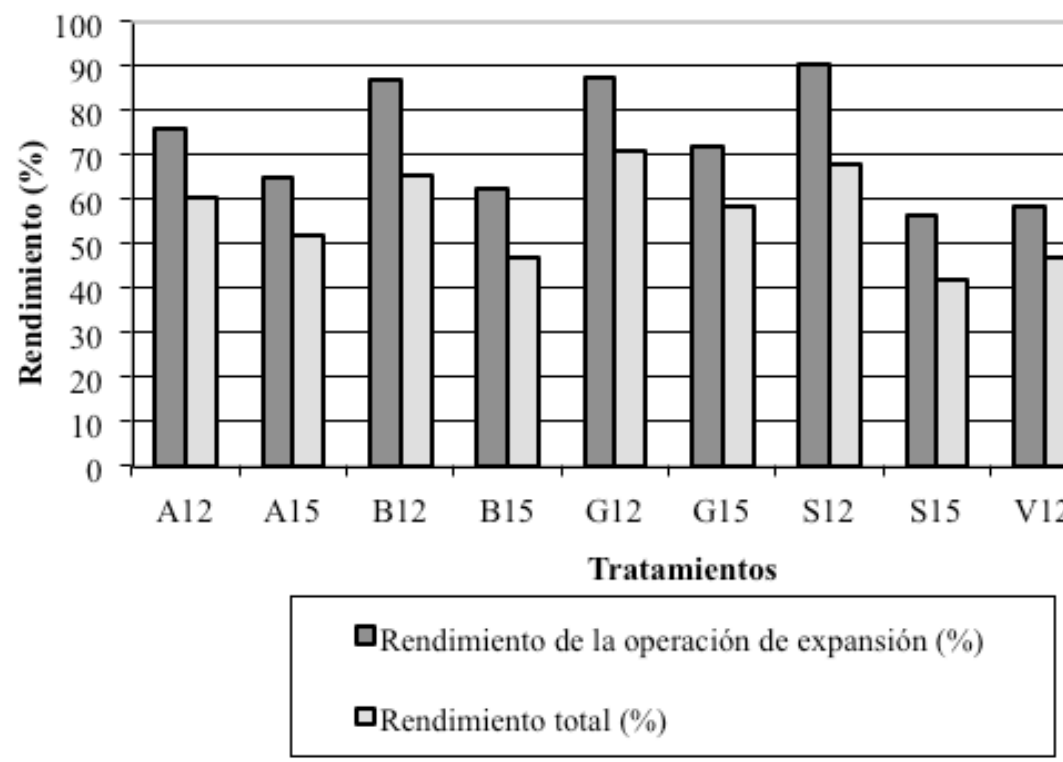

Figura 4. Rendimiento de la operación de expansión y rendimiento total para los diferentes tratamientos.

variedades Gergona $(1,4)$, Señorita $(1,39)$ y Blanca $(1,31)$, acondicionadas a $12 \%$ de humedad inicial mientras que las variedades con menor IE fueron la Amarilla $(1,0)$ y la Verde $(1,01)$, acondicionadas a $15 \%$ de humedad inicial (Fig. 4).

No se encontró ninguna relación (directa o inversa) entre el contenido de almidón y el índice de expansión de las diferentes variedades de habas. Sin embargo, Shuh y Tsai (citados en Hoke, Houska, Pruchova, Gabrovska, Vaculova y Paulickova, 2005), indican que no es sólo el valor del contenido de almidón el que influye en el índice de expansión del grano, sino también su composición. Según esto, un grano con alto contenido de amilosa y baja amilopectina, no es apropiado para esta tecnología. Según Badui (1999), el almidón con alto contenido de amilopectina y baja amilasa gelatiniza más rápido, esto permite que se formen burbujas más grandes en el corto tiempo que dura la operación de expansión.

Para corroborar si el contenido de amilosa y amilopectina influyeron en el índice de expansión, se realizó una prueba de Correlación de Pearson. Los resultados de esta indicaron que el índice de expansión de las variedades de habas estudiadas no está asociado al contenido de almidón ni al contenido de amilosa y amilopectina. Se puede ver que las variedades Gergona $(1,4)$, Señorita $(1,39)$ y Blanca $(1,31)$ tuvieron los IE mayores, acondicionadas a $12 \%$ de humedad inicial, mientras que las variedades que presentaron un menor IE fueron la Verde (1,01$1,06)$ y la Amarilla $(1,0-1,07)$ acondicionadas a $12 \%$ y $15 \%$ de humedad inicial, respectivamente. La variedad que presentó un mayor contenido de amilopectina fue la variedad Señorita $(55,86 \%)$.

\section{Evaluación del rendimiento}

En la Tabla 5 se puede ver el rendimiento de la operación de expansión, el rendimiento total para cada tratamiento y la humedad final del producto expandido así como la humedad final del producto y en la Fig. 4 se encuentran graficados los rendimientos de manera comparativa. Teniendo en cuenta los tratamientos con $12 \%$ de humedad inicial, la variedad que obtuvo un mayor rendimiento en la operación de expansión fue la Señorita $(90,60 \%)$, sin embargo la humedad final fue de 7,25\%, ligeramente superior a lo recomendado por Hurtado (1986), quien 
Tabla 5. Rendimiento de la operación de expansión, rendimiento total y contenido de humedad para los diferentes tratamientos (\%)

\begin{tabular}{cccc}
\hline Tratamiento & $\begin{array}{c}\text { Rendimiento de la } \\
\text { expansión }\end{array}$ & Rendimiento total & $\begin{array}{c}\text { Humedad final del } \\
\text { producto expandido }\end{array}$ \\
\hline A12 $\left(^{*}\right)$ & 76,09 & 60,36 & 6,37 \\
A15 & 65,22 & 51,74 & 8,95 \\
B12 & 87,14 & 65,56 & 3,31 \\
B15 & 62,73 & 47,20 & 5,84 \\
G12 & 87,66 & 71,05 & 7,01 \\
G15 & 71,97 & 58,33 & 9,13 \\
S12 & 90,60 & 67,75 & 7,25 \\
S15 & 56,32 & 42,11 & 8,17 \\
V12 & 58,37 & 46,81 & 2,72 \\
V15 & 59,21 & 47,48 & 8,23 \\
\hline
\end{tabular}

Nota: $(*)$ Variedad y \% de humedad al ingreso del proceso de expansión.

indica que lo ideal es entre 3-7 \%. Las variedades que obtuvieron valores bajos de humedad final fueron las habas Blanca y la Verde, ambas ingresaron al proceso con $12 \%$ de humedad inicial, con rendimientos de $65,56 \%$ y $46,81 \%$ respectivamente.

El Haba Señorita, que ingresó al proceso de expansión con $15 \%$ de humedad inicial, obtuvo el menor rendimiento tanto en el proceso de expansión $(56,32 \%)$ como en el proceso en total $(42,11 \%)$, esto se debe a que presentaron una gran cantidad de granos quemados y partidos que fueron desechados.

\section{Determinación del color}

En la Tabla 6 se muestran los promedios de los valores de "L", "a" y "b" para todos los tratamientos. Los valores de "a" y "b" corresponden a los colores rojo y amarillo respectivamente ya que en todas las muestras son valores positivos (Konica Minolta, 2003). Se encontró que para todas las variedades, excepto la variedad Gergona, los valores de "a" y "b" son más altos en los acondicionados a $15 \%$ de humedad inicial que a los acondicionados a 12 $\%$. Esto indica que estas muestras tienen una coloración más saturada y vívida, mientras que las variedades con 12 $\%$ tendrían una coloración menos saturada y más grisácea. La combinación de estas diferentes tonalidades de amarillo y rojo producen diferentes tonos de marrón para cada uno de los tratamientos. El color marrón observado se debe en parte a la caramelización de los carbohidratos del grano que ocurre durante el calentamiento de éste (Desrosier, 1993). Otro motivo para el oscurecimiento de los granos de habas es la reacción de Maillard que implica la interacción entre azúcares y aminoácidos (Braverman, 1976).

En cuanto a la brillantez, tres de las cinco variedades de habas mostraron valores de "L" mayores en los tratamientos con $15 \%$ de humedad que en los tratamientos con $12 \%$ de humedad, representando una mayor palidez o brillantez del color (Konica Minolta, 2003). Según esto, los colores fueron más brillantes o pálidos a $15 \%$ de humedad en el caso de las habas Blanca, Gergona y Señorita. Esto se debe en gran medida a que con $15 \%$ de humedad en el grano, éste no se quema tanto como a $12 \%$ de humedad.

\section{Evaluación sensorial}

Prueba de Ranking: el grado de aceptación del consumidor hacia las diferentes variedades de habas expandidas, de mayor aceptación a menor aceptación, fue el siguiente: Blanca, Verde, Gergona, Señorita, Amarilla. Según los resultados de la prueba de Friedman se concluyó que existía evidencia estadística a un nivel de significación de $95 \%$ para decir que al menos uno de los grados de aceptación del consumidor de una variedad de haba expandida se diferencia significativamente del resto.

Comparaciones Múltiples: tanto el Haba Blanca como la Verde fueron consideradas agradables para el consumidor, no habiendo diferencia significativa en el grado de aceptación de ambas, pero sí se encontró que diferían de las variedades Gergona, Señorita y Amarilla.

Categorización cuantitativa relativa: en todos los casos se encontró que no existían diferencias significativas entre los jueces, pero sí entre los tratamientos (a un nivel de significancia de 0,05). Los resultados se sometieron a un análisis de varianza (ANVA) siguiendo un diseño de bloques completamente al azar (DBCA), donde se determinó que al menos una de las variedades de habas presenta diferencias significativas en el color con respecto a otra variedad, debido a esto los resultados se sometieron a las comparaciones múltiples con la prueba de Duncan y según los resultados obtenidos existe suficiente evidencia estadística, a un nivel de significancia de 95\%, para afirmar que: a) existen diferencias de color entre todas las variedades excepto entre las variedades Blanca y Amarilla y entre la Gergona y la Verde, siendo estas dos últimas las más aceptadas; b) el olor de la variedad Amarilla difiere de las demás variedades, mientras que la Blanca difiere de todas las variedades menos de la Verde, siendo esta última 
Tabla 6. Dimensiones "L", “a" y "b" del color de las habas expandidas. Expansión de las habas evaluadas

\begin{tabular}{ccccccc}
\hline & $12 \%$ Humedad inicial & \multicolumn{3}{c}{$15 \%$ Humedad inicial } \\
& $\mathrm{a}$ & $\mathrm{b}$ & $\mathrm{L}$ & $\mathrm{a}$ & $\mathrm{b}$ & $\mathrm{L}$ \\
\hline Var. Amarilla & 5,43 & 14,65 & 56,44 & 5,46 & 14,88 & 49,10 \\
Var. Blanca & 5,19 & 12,89 & 52,79 & 5,46 & 14,05 & 54,40 \\
Var. Gergona & 7,69 & 13,45 & 52,90 & 4,77 & 12,34 & 55,41 \\
Var. Señorita & 5,76 & 11,43 & 48,49 & 7,24 & 12,76 & 51,30 \\
Var. Verde & 6,84 & 15,14 & 58,23 & 7,94 & 9,28 & 48,76 \\
\hline
\end{tabular}

*Open RGB Versión 2.01.80406. Copyright (C) 2008, Logicol.

Tabla 7. Comparación de la composición químicoproximal del Haba Blanca expandida con las habas fritas comerciales $(\mathrm{g} / \mathbf{1 0 0} \mathrm{g})$.

\begin{tabular}{ccc}
\hline Componente & $\begin{array}{c}\text { Haba Blanca } \\
\text { expandida }\end{array}$ & $\begin{array}{c}\text { Haba frita } \\
\text { comercial } \\
\text { "Carter" }\end{array}$ \\
\hline Humedad & 3,31 & $*$ \\
Carbohidratos & 61,89 & 54 \\
Proteína & 27,45 & 27,17 \\
Grasa & 2,56 & 12,12 \\
Fibra & 1,48 & $*$ \\
Ceniza & 3,31 & $*$ \\
\hline
\end{tabular}

la que obtuvo la mejor calificación, colocándose por encima de lo ideal; c) existen diferencias significativas de sabor entre la variedad Blanca y la Amarilla, la Verde y la Señorita, pero no con la Gergona. El Haba Blanca tuvo un puntaje bastante cercano a lo ideal. El sabor de las Habas Verde y Señorita fue calificado como ligeramente más fuerte que la Blanca, mientras que el sabor de la Gergona y la Amarilla fue considerado muy suave.

Finalmente, los resultados para la textura se evaluaron mediante la prueba no paramétrica de Friedman donde se determinó que al menos una de las variedades presentó textura diferente a las demás. Las pruebas comparativas dieron como resultado que existe suficiente evidencia estadística a un nivel de significancia de $95 \%$ para afirmar que la variedad Blanca no presenta diferencias significativas en cuanto a su textura con respecto a la Gergona, Señorita y Verde; así como la Señorita no presenta diferencias significativas con respecto a la Gergona. Las Habas Gergona y Amarilla fueron calificadas como de textura muy suave, mientras que la textura del haba Verde fue calificada como muy dura.

Perfil de textura: Según los resultados de los análisis fisicoquímicos y sensoriales anteriores, se tomó como tratamiento ganador al Haba Blanca con $12 \%$ de humedad inicial. A este producto se le realizó un perfil de textura para compararlo con un producto comercial de habas fritas de la marca "Carter". Inicialmente, se determinó que la percepción de los jueces no incide en la puntuación de las características de crocantez, dureza, gomosidad, adhesividad ni arenosidad (se determinó mediante una evaluación estadística en un diseño no paramétrico para la prueba de Kruskal Wallis, dando negativo a un nivel de significación de 0,05).

La comparación individual de las características de ser crocantes, duras, adhesivas y gomosas se realizó mediante análisis de varianza en diseños DBCA, mientras que el ser arenosas fue comparada mediante la prueba alternativa de Friedman.

El análisis estadístico a un nivel de significación de 0,05, dio como resultado que no existían

diferencias significativas entre las habas expandidas y las habas fritas comerciales en cuanto a dureza, la cual es uno de los aspectos más importantes ya que muchas veces las habas tostadas son rechazadas por este factor, pero sí se encontraron diferencias significativas en que sean crocantes, arenosas y en la adhesividad y gomosidad del producto.

Las habas expandidas no demostraron ser tan crocantes como las habas fritas, debido a la gomosidad más alta que presentó el producto expandido. Esto a la vez, se ve traducido en la mayor adhesividad a los dientes que presentaron las habas expandidas al compararlas con las habas fritas comerciales.

\section{Análisis proximal}

Se realizó el análisis químico-proximal de la variedad de Haba Blanca, considerada la más adecuada para el proceso de expansión por explosión y se comparó con una muestra de haba frita comercial (Tabla 7). Según Nath, Chattopadhyay y Majumdar (2006), el consumidor moderno cada vez exige más productos de rápida preparación o listos para el consumo, pero que sean saludables. El aumento de la preocupación por enfermedades degenerativas y la búsqueda de la información por parte del consumidor común han hecho que se cree una conciencia nutricional que apunta al consumo de alimentos saludables. Según Nath, A., Chattopadhyay, P. y Majumdar, G. (2006), el consumidor moderno cada vez exige más productos de rápida preparación o listos para el consumo, pero que sean saludables, de otro lado Singh et al. (citado por Nath et al., 2006) indica que los snacks fritos presentan un alto contenido de grasa que es dañino para el corazón humano y que el alto contenido de aceite propicia que el producto se ponga rancio, haciendo que su vida en anaquel sea corta, por lo que se tiene que realizar una operación de empacado 
complicada que eleva el costo unitario del producto (Nath et al, 2006). Se encontró que el contenido de grasa de las habas fritas $(12,12 \%)$ fue 4,7 veces más que el contenido de grasa de las habas expandidas $(2,56 \%)$.

\section{Conclusiones}

La variedad Señorita tuvo el mayor contenido de proteínas $(25,4 \%)$ y de almidón (16,78\%), pero el menor valor de amilosa $(44,14 \%)$, mientras que el menor valor de proteína fue de la variedad Verde $(22,92 \%)$ y la variedad Blanca tuvo el mayor contenido de carbohidratos $(53,45$ $\%$, siendo la variedad de Haba Blanca la más adecuada para el proceso de expansión por explosión.

Existen diferencias significativas entre el IE de las habas que ingresan al proceso de expansión con $12 \%$ y $15 \%$ de humedad respectivamente, siendo los mayores valores cuando ingresan con $12 \%$. La variedad con mayor índice de expansión fue la Gergona $(1,4)$, seguida por las variedades Señorita $(1,39)$, Blanca $(1,31)$, Amarilla $(1,07)$ y Verde $(1,06)$. Sin embargo, los índices de expansión obtenidos para las habas de estas cinco variedades fueron menores que los obtenidos con el trigo $(8-16)$ y el arroz $(6-8)$.

Al comparar la textura del haba expandida de la variedad Blanca con el haba frita de la marca "Carter" se pudo ver que la dureza y lo arenoso del producto fue similar para el haba expandida y el haba frita comercial, lo crocante fue mayor para el haba frita, la gomosidad se percibió más en el haba expandida y finalmente la adhesividad en los dientes fue ligeramente mayor para el haba expandida.

\section{Literatura citada}

La asociación de las comunidades analíticas (AOAC). 1995. Official methods of analysis of AOAC International. EUU: Association of Official Analytical Chemists.

Callejo, M. 2002. Industrias de Cereales y derivados. Madrid, España: AMV Ediciones.

Badui, S. 1999. Química de los alimentos. Naucalpán de Juárez, México: Addison Wesley Longman, S.A. de C.V.

Braverman, J. 1976. Introducción a la Bioquímica de los alimentos. D.F., México: El Manual Moderno, S.A. de C.V.

Capcha, A. 1977. Evaluación de la aptitud combinatoria general mediante Top Cross y determinación de la capacidad y volumen de expansión de líneas S1 en Maíz Reventón PMV-273 (Zea Mays everta) (Tesis). Universidad Nacional Agraria La Molina, Perú.

Cenzano, E. 1996. Extracción y caracterización de los almidones de tres clones de achira (Tesis). Universidad Nacional Agraria La Molina, Perú.

Desrosier, N. 1993. Conservación de alimentos. Segunda edición. México: Compañía Editorial Continental, S.A. de C.V.

Hoke, K.; Houska, M.; Pruchova, J.; Gabrovska, D.; Vaculova, K. y Paulickova, I. 2005. Optimization of puffing naked barley. Journal of Food Engineering. República Checa, 80: 1016-1022.

Huaringa, A.; Camarena, F.; Chiappe, L. y Mostacero, E. 2003. Manual del cultivo de Haba. Lima, Perú: Universidad Nacional Agraria La Molina.

Hurtado, F. 1986. Productos expandidos con el cañón esponjador. Taller Feria: "Desarrollo de la pequeña y mediana industria alimentaria en Latinoamérica". Perú.

Instituto Nacional de Estadística e Informática (INEI). 2011. Desnutrición crónica se redujo en 4.7 puntos porcentuales entre los años 2007 al 2010. INEI. Disponible en: http://www.inei.gob.pe/web/NotaPrensa/ Attach/11978.pdf

Konica Minolta. 2003. Precise color Communications. Konica Minolta Sensing, Inc. Konica Minolta. Recuperado de http://www2.konicaminolta.eu/eu/Measuring/pcc/es/ index.html

Lusas, E. y Rooney, L. 2001. Snack Foods Processing. Technomic Publishing Company Inc. EEUU.

Mc Grance, S.; Cornell, H. y Melbourne, C. 1998. A Simple and Rapid Colorimetric Method for the Determination of Amylose in Starch Products. Starch Magazine, 4: 158-163.

Ministerio de Agricultura (MINAG). 2010. Dinámica Agropecuaria. Oficina de Estudios Económicos y Estadísticos, Perú: MINAG. Disponible en: http:// www.minag.gob.pe/download/pdf/especiales/dinamica/ dinamicaagropecuaria1997-2009.pdf

Nath, A.; Chattopadhyay, P. y Majumdar, G. 2006. High temperature short time air puffed ready-to-eat (RTE) potato snacks: Process parameter optimization. Journal of Food Engineering, 80: 770-780.

Ureña, M.; D’Arrigo, M. y Girón, O. 1999. Evaluación Sensorial de los Alimentos. Aplicación Didáctica. Perú: Editorial Agraria.

Zapotoczny, P.; Markowski, M.; Majewska, K.; Ratajski, A. y Konopko, H. 2004. Effect of temperature on the physical, functional, and mechanical characteristics of hot-air-puffed amaranth seeds. Journal of Food Engineering, 76: 469-476. 